\title{
3D Clinical Evaluation of Unusual Anatomy of A Maxillary Second Molar: A Case Report
}

\author{
Dario Di Nardo*1, Gianluca Gambarini ${ }^{1}$, Riccardo Costantini ${ }^{1}$, Luca Testarelli ${ }^{1}$, Lucila Piasecki ${ }^{2}$ and Dina \\ Al-Sudani ${ }^{3}$ \\ ${ }^{1}$ Department of Oral and Maxillo Facial Sciences, Sapienza University of Rome, Italy
}

${ }^{2}$ Department of Periodontics and Endodontics, University at Buffalo, USA

${ }^{3}$ Department of Restorative Dental Sciences, College of Dentistry, King Saud University, Saudi Arabia

Received: January 04, 2018; Published: January 10, 2018

*Corresponding author: Dott Dario Di Nardo, Department of Oral and Maxillo Facial Sciences, Sapienza University of Rome, Italy, Tel: 39 3393935527;

Email: dario.dinardo@uniroma1.it

\section{Abstract}

Aim To present the combined use of CBCT images and new endodontic software to identify and then facilitate the location and treatment of a canal in an unusually placed second mesio-buccal root of a maxillary left second molar. Summary A 45-year-old female was referred for root canal treatment on her maxillary left second molar tooth (tooth 27). Due to superimposition of the third molar and the position of the tooth that made conventional radiographs unhelpful, a pre-operative limited FOV, Low-Dose Technology ${ }^{\mathrm{TM}}$ CBCT image was taken with an OP-300 Maxio ${ }^{\mathrm{TM}}$ device (Instrumentarium, Helsinki, Finland). The CBCT images were then imported and analyzed using 3D Endo ${ }^{\mathrm{TM}}$ Software (Dentsply Sirona, Wels bei Salzburg, Austria). Within the software, the canal orifices and foramina were located and identified on the display, which allowed the software to automatically reveal and measure the pathway of each canal, which was traced and viewed in both frontal and mesial perspectives. The images revealed the presence of a separate second mesiobuccal root that contained one canal. Root canal treatment was then performed using the information derived from the software. The orifice of the canal in the second MB root was located in an unusual position: $5.99 \mathrm{~mm}$ palatally to the main MB canal and $7.57 \mathrm{~mm}$ medially from the palatal canal. An immediate post-operative limited FOV, low-dose CBCT was taken to assess the quality of the root filling. After one year the tooth was asymptomatic and radiographic examination revealed no sign of periapical pathosis.

Keywords : Anatomy, Maxillary Molar, Mesio-Buccal Canal, CBCT

Key Learning Points:
a)
The variable anatomy of maxillary second molar teeth
b) The unusual (palatal) location of the second mesio-buccal root and canal in this case
c) The combined use of CBCT and 3D endodontic software for pre-operative assessment of complex cases in order to identify and manage risk.

\section{Introduction}

The variability and complexity of root canal morphology in multi-rooted teeth is a challenge for the practitioner [1]. Knowledge of the most common anatomic characteristics and their possible variations is fundamental, because a missed canal is a leading cause of treatment failure $[2,3]$. The anatomy of maxillary second molars is similar to that of maxillary first molars; however, their main distinctive morphological feature is that the usual three roots align more closely and are occasionally fused [4]. Although four canals are found less often in the second molar than in the first molar [5]. Its canal configuration is more variable [6]. Indeed, many studies have described morphologic variations in maxillary molars, with multiple canals in each root, especially bi- or tri-furcated palatal canals [7-11]. Unusual anatomy in maxillary second molars is often difficult to diagnose because of its posterior location [12]. The superimposition of anatomical structures on conventional radiographs of this tooth may fail to reveal a bifurcated palatal root canal or other anatomical variations, such as single or double roots and fused roots $[13,14]$. Cone-beam computed tomographic (CBCT) images are useful in providing accurate anatomic details in three dimensions for diagnosis and treatment planning before root canal treatment $[15,16]$. More recently, a software package has been developed for analyzing endodontic parameters on CBCT images, 3D Endo ${ }^{\mathrm{TM}}$ (Dentsply Sirona, Wels bei Salzburg, Austria), which provides a user-friendly interface for the identification, 
visualization and measurement of root canals using a colour coded system for enhanced visualization of the trajectory of each canal.

\section{Case Report}

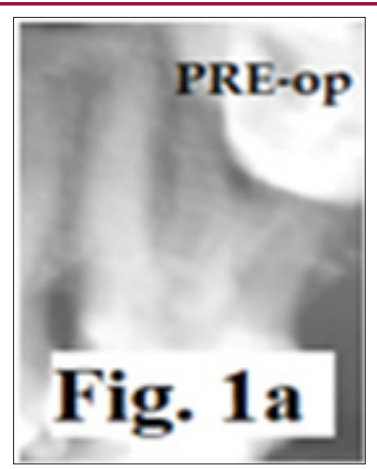

Figure 1a: Pre operative traditional radiograph taken by referring dentist with superimposition of the third molar.

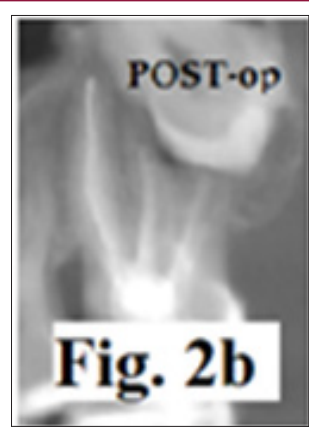

Figure 1b: Control after one year: traditional radiograph taken by referring dentist.

A 45-year-old female was referred for root canal treatment on her maxillary left second molar (tooth 27). The referring dentists had previously taken an intraoral digital radiograph (Figure 1a) but due to the superimposition of the third molar and position of the tooth a pre-operative limited FOV, Low-Dose Technology ${ }^{\mathrm{TM}}$ CBCT image was acquired using an 0P-300 Maxio $^{\mathrm{TM}}$ device (Instrumentarium, Helsinki, Finland) adopting the following parameters: field of view (FOV): $50 \times 50 \mathrm{~mm}$, spatial resolution: 0.28 mm voxels, $90 \mathrm{kVp}$ and $3.2 \mathrm{~mA}$. A cold pulp sensibility test initiated a response from the patient. No swelling, mobility or tenderness to percussion was recorded. The CBCT images revealed a very unusual anatomy with a second mesial root located palatally. This root was adjacent to the main mesial root in the coronal region, and then diverged palatally; it had its own canal with an independent foramen (Figure 2a) similar to the MB2 canal that would be present in a single mesio-buccal root. To visualize better this unusual configuration and to determine the optimum access cavity design so as to locate all canal orifices, CBCT images were then analyzed using the 3D Endo $^{\mathrm{TM}}$ Software. The software was used to crop the area corresponding to the tooth in the axial and para-sagittal planes. Once the tooth was selected, the canal orifices and foramina were located and identified in the next screen. This allowed the software to automatically reveal the pathways of the four canals, which were traced in different colours and viewed in frontal and mesial views (Figure $2 \mathrm{~b}$ ). The software also allowed a virtual access cavity design to be visualised along with the path of insertion of endodontic instruments within the canals (Figures 3a-3b); the number of roots and canals, their lengths and trajectories, and the position of orifices were identified easily. 3D volumetric rendering of the tooth was then performed using the OP-300 Maxio $^{\mathrm{TM}}$ imaging software, to compare 3D pre- and post-operative images (Figures $4 a-4 b)$.

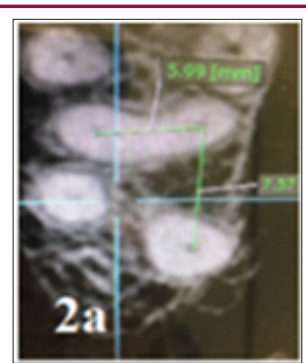

Figure 2a: Axial CBCT image showing distance between second MB canal and MB (5,99 $\mathrm{mm})$ and between second $\mathrm{MB}$ canal and palatal canal $(7,57 \mathrm{~mm})$.

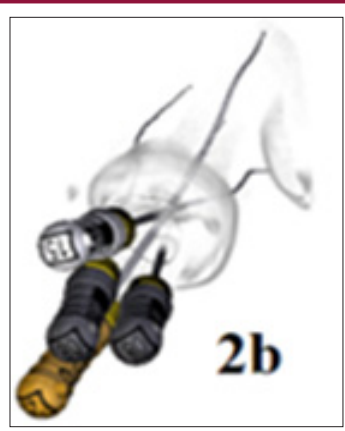

Figure 2b: 3D Endo ${ }^{\mathrm{TM}}$ Software image showing virtual access cavity and insertion of endodontic instruments in the four canals.

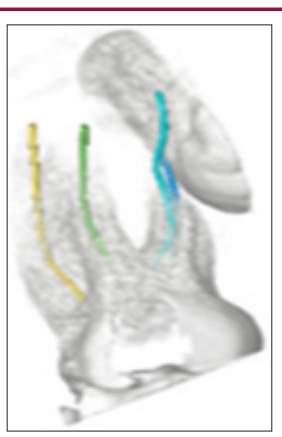

Figure 3a: Images from 3D Endo ${ }^{\mathrm{TM}}$ Software showing the different canal trajectories with different colours.

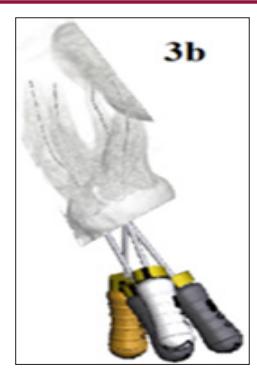

Figure 3b: Virtually inserted endodontic instruments inside the four different canals and following their trajectories. 


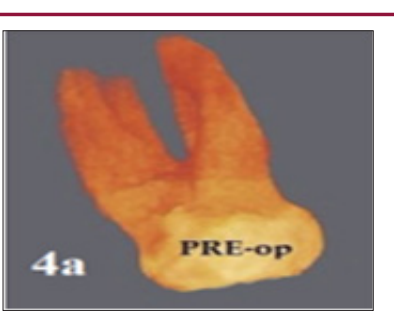

Figure 4a: Preoperative 3D reconstruction (rendering) of initial anatomy (4a) showing the unusual root anatomy.

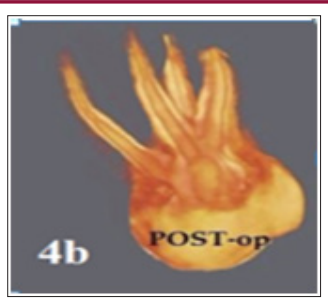

Figure 4b: Postoperative 3D rendering after canal obturation showing the four canals properly treated.

Root canal treatment was performed, using NiTi rotary instruments and a warm gutta-percha filling technique. The canal in the unusual second mesial root was located, negotiated and treated. The canal lengths estimated by the software were confirmed using an apex locator (Apex ID, Kerr Endodontics, Orange, Ca, USA). No intra-operative radiograph was taken. A CBCT post-operative limited-FOV low-dose image, using the same parameters, was taken to assess the quality of the root filling and to ensure the entire canal system was treated (Figure $4 \mathrm{~b}$ ). The access cavity was restored using a flowable, high viscosity, bulk composite (SonicFill, Kerr, Bioggio, Switzerland). After one year the patient remained asymptomatic. A digital radiograph (Gendex GXPS-500, Kavo, Biberach, Germany) taken by the referring dentist did not reveal any signs of periapical pathosis (Figure 1b).

\section{Discussion}

A high proportion of treatment failures in maxillary molars occurs due to the impossibility of locating, instrumenting and filling the second MB canal $[17,18]$. With the result that many studies have evaluated the presence and location of this canal [2,19-21]. Interestingly, no reports on the actual treatment of a second $\mathrm{MB}$ root have been published. In the present case, a second mesial root with its own canal was detected in an unusual palatal position (Figure 2a); such a palatal orifice could have been missed without the use of 3D imaging. The CBCT images and 3D Endo ${ }^{\mathrm{TM}}$ Software facilitated the rapid detection of this key morphological feature and allowed the ideal access cavity design for locating and treating all four canals to be visualised pre-operatively. Each CBCT image was equivalent to half the radiation dose compared to an intraoral nondigital radiograph [22]. The 3D rendered images clearly revealed that this root was fused to the main MB root in the coronal region and that the canals in each root had an independent foramen (Figure. 3-4). Post-operative 3D rendered images confirmed the four canals had been shaped and filled, in a more detailed and clear way than traditional radiographs (Figure 1b) For maxillary molars, the usual way of assessing tooth anatomy in case reports has been via periapical radiographs, whereas the more popular method in morphological studies has been CBCT [4]. In a CBCT study [24]. The second MB canal was identified in $48 \%$ of the cases, and it was located $2.2 \pm 0.54 \mathrm{~mm}$ palatally and $0.98 \pm 0.35$ mesially to the first MB canal. In another study, it was located $2 \mathrm{~mm}$ palatally and $1 \mathrm{~mm}$ mesially to the first MB canal [5]. In the present case, the orifice of the canal in the second mesial root was located in a very unusual position: $5.99 \mathrm{~mm}$ palatally ( $0.3 \mathrm{~mm}$ mesially) to the main $\mathrm{MB}$ canal orifice and $7.57 \mathrm{~mm}$ mesially from the palatal canal (Figure 2a). On the contrary, the MB, DB and palatal canal orifices were located in the usual position for maxillary molars. Second MB canals can often be managed successfully with the aid of a dental operating microscope [25]. However, the search for its orifice is usually limited to a few millimetres palatally and mesially from the main MB canal: the rationale being to avoid unnecessary weakening of the mesial marginal ridge and palatal cusp. In the present case, an intraoral radiograph did not provide sufficient information for the identification and location of the second mesial root and canal, due to the superimposition of anatomical structures and the impacted third molar.

\section{Conclusion}

The use of low-dose CBCT images and new 3D Endo ${ }^{\mathrm{TM}}$ Software, allowed rapid, simple and precise preoperative assessment of the second mesial root and canal, thus increasing the quality and outcome of the treatment by avoiding further complications related to a missed root and canal.

\section{References}

1. Fakhari E, Shokraneh A (2013) A Maxillary Second Molar with Two Separate Palatal Roots: A Case Report. Journal of Dentistry 14(2): 87-89.

2. Blattner TC, George N, Lee CC, Kumar V, Yelton CD (2010) Efficacy of cone-beam computed tomography as a modality to accurately identify the presence of second mesiobuccal canals in maxillary first and second molars: a pilot study. Journal of Endodontics 36(5): 867-870.

3. Eskandarinezhad M, Ghasemi N (2012) Nonsurgical endodontic retreatment of maxillary second molar with two palatal root canals: a case report. Journal of Dental Research, Dental Clinics, Dental Prospects 6(2): 75-78.

4. Ghasemi N, Rahimi S, Shahi S, Samiei M, Frough Reyhani M, et al.(2017) A Review on Root Anatomy and Canal Configuration of the Maxillary Second Molars. Iranian Endodontic Journal Winter 12(1): 1-9.

5. Zhang R, Yang H, Yu X, Wang H, Hu T, et al. (2011) Use of CBCT to identify the morphology of maxillary permanent molar teeth in a Chinese subpopulation. International Endodontic Journal Feb;44(2), 162-169.

6. Alrahabi M, Sohail Zafar M (2015) Evaluation of root canal morphology of maxillary molars using cone beam computed tomography. Pakistan Journal of Medical Sciences 31(2): 426-430.

7. Baratto-Filho F, Fariniuk LF, Ferreira EL, Pecora JD, Cruz-Filho AM, et al.(2002) Clinical and macroscopic study of maxillary molars with two palatal roots. International Endodontic Journal 35(9): 796-801.

8. Barbizam JV, Ribeiro RG, Tanomaru Filho M (2004) unusual anatomy of permanent maxillary molars. Journal of Endodontics 30(9): 668-671.

9. Holderrieth S, Gernhardt CR (2009) Maxillary molars with morphologic variations of the palatal root canals: a report of four cases. Journal of Endodontics 35, 1060-1065.

10. Qun L, Longing N, Qing Y, Yuan L, Jun W, et al. (2009) A case of asymmetric maxillary second molar with double palatal roots. Quintessence International 40(4): 275-276. 
11. Paul B, Dube K (2013) Endodontic treatment of a maxillary second molar with two separate palatal roots: a case report. Journal of Clinical and Diagnostic Research 7(12): 3127.

12. Nosrat A, Verma P, Hicks ML, Schneider SC, Behnia A, et al.(2017) Variations of Palatal Canal Morphology in Maxillary Molars: A Case Series and Literature Review. Journal of Endodontics 3(11):1888-1896

13. Alani AH (2003) Endodontic treatment of bilaterally occurring 4-rooted maxillary second molars: case report. Journal of the Canadian Dental Association 69(11): 733-735.

14. Neelakantan P, Subbarao C, Ahuja R, Subbarao CV, Gutmann JL (2010) Cone-beam computed tomography study of root and canal morphology of maxillary first and second molars in an Indian population. Journal of Endodontics 36(10): 1622-1627.

15. Domark JD, Hatton JF, Benison RP, Hildebolt CF (2013) An ex vivo comparison of digital radiography and cone-beam and micro computed tomography in the detection of the number of canals in the mesiobuccal roots of maxillary molars. Journal of Endodontics 39(7): 901-905.

16. Durack C, Patel S (2012) Cone beam computed tomography in endodontics. Brazilian Dental Journal 23(3): 179-181.

17. Vizzotto MB, Silveira PF, Arus NA, Montagner F, Gomes BP, et al. (2013) CBCT for the assessment of second mesiobuccal (MB2) canals in maxillary molar teeth: effect of voxel size and presence of root filling. International Endodontic Journal 46(9): 870-876.

18. Wolcott J, Ishley D, Kennedy W, Johnson S, Minnich S, et al. (2005) A 5 yr clinical investigation of second mesiobuccal canals in endodontically treated and retreated maxillary molars. Journal of Endodontics 31(4): 262-264.

19. Arora A, Acharya SR, Saraswathi MV, Sharma P, Ather A (2013) Dilemmas pertaining to three canals in the mesiobuccal root of a maxillary second molar: a case report. Restorative Dentistry \& Endodontics 38(3): 172 177.
20. Georgia NE, Taxiarchis KG, Nikolaos KP (2015) Evaluation of the Root and Canal Morphology of Maxillary Permanent Molars and the Incidence of the Second Mesiobuccal Root Canal in Greek Population Using Conebeam Computed Tomography. Open Dentistry Journal 9:267-272.

21. Reis AG, Grazziotin-Soares R, Barletta FB, Fontanella VR, Mahl CR (2013) Second canal in mesiobuccal root of maxillary molars is correlated with root third and patient age: a cone-beam computed tomographic study. Journal of Endodontics 39(5): 588-592.

22. Silveira LF, Marques MM, da Costa RK, Martos J, Lorenzi A (2013) Location and negotiability of second mesiobuccal canal in upper molar by tomographic and anatomical macroscopic analysis. Surgical and Radiological Anatomy 35(9), 791-795.

23. Ludlow JB, Timothy R, Walker C (2015) Effective dose of dental CBCT-a meta analysis of published data and additional data for nine CBCT units. Dentomaxillofacial Radiology 44(1): 20140197.

24. Betancourt P, Navarro P, Cantin M, Fuentes R (2015) Cone-beam computed tomography study of prevalence and location of MB2 canal in the mesiobuccal root of the maxillary second molar. International Journal of Clinical and Experimental Medicine 8(6): 9128-9134.

25. Suresh M, Karthikeyan K, Mahalaxmi S (2017) Maxillary Second Molar with Fused Root and Six Canals- A Case Report. Journal of Clinical and Diagnostic Research Apr 11(4): ZD35-ZD37.

26. Kim Y, Lee SJ, Woo J (2012) Morphology of maxillary first and second molars analyzed by cone-beam computed tomography in a korean population: variations in the number of roots and canals and the incidence of fusion. Journal of Endodontics 38(8): 1063-1068.

27. Holderrieth S, Gernhardt CR (2009) Maxillary molars with morphologic variations of the palatal root canals: a report of four cases. Journal of Endodontics 35: 1060-1065.

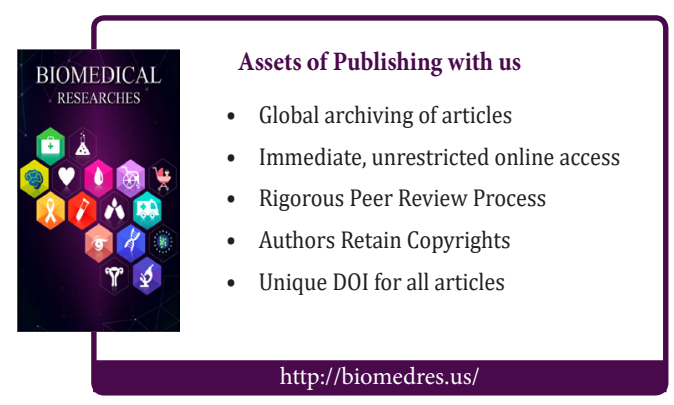

